\title{
Correction to: Deep reinforcement learning for multi-objective placement of virtual machines in cloud datacenters
}

\author{
Luca Caviglione $^{1}$ (D) Mauro Gaggero $^{2}$ (D) $\cdot$ Massimo Paolucci $^{3}$ (D) $\cdot$ Roberto Ronco $^{3}$ (D)
}

Published online: 15 January 2021

(c) The Author(s) 2021

\section{Correction to: Soft Computing} https://doi.org/10.1007/s00500-020-05462-x

Page 2: Column 2, lines 2-4, previously read: "Specifically, we consider a decision maker that, after a proper training, is able to select the most suitable heuristic for compute the placement for each VM requested by end users."

Should read: "Specifically, we consider a decision maker that, after a proper training, is able to select the most suitable heuristic to compute the placement for each VM requested by end users."

Page 2: Column 2, paragraph 2, lines 4-8, previously read: To evaluate its effectiveness, a comparisons against solutions widely adopted in the literature and real-world scenarios are presented, including the use of workload traces collected in a production-quality cloud datacenter.

Should read: To evaluate its effectiveness, comparisons against solutions widely adopted in the literature and real-world scenarios are presented, including the use of workload traces collected in a production-quality cloud datacenter.

The original article can be found online at https:// doi.org/10.1007/s00500-020-05462-x.

Massimo Paolucci

massimo.paolucci@unige.it

Luca Caviglione

luca.caviglione@ge.imati.cnr.it

Mauro Gaggero

mauro.gaggero@cnr.it

Roberto Ronco

roberto.ronco@edu.unige.it

1 IMATI, National Research Council of Italy, Via De Marini 6, 16149 Genoa, Italy

2 INM, National Research Council of Italy, Via De Marini 6, 16149 Genoa, Italy

3 DIBRIS, University of Genoa, Via All'Opera Pia 13, 16145 Genoa, Italy
Page 4: Column 1, paragraph 2, lines 2-5, previously read: In more detail, users produce a workload composed of new VM requests, which are collected by the Admission \& placement module.

Should read: In more detail, users produce a workload composed of new VM requests, which are collected by the "Admission \& placement" module.

The original article has been corrected.

Open Access This article is licensed under a Creative Commons Attribution 4.0 International License, which permits use, sharing, adaptation, distribution and reproduction in any medium or format, as long as you give appropriate credit to the original author(s) and the source, provide a link to the Creative Commons licence, and indicate if changes were made. The images or other third party material in this article are included in the article's Creative Commons licence, unless indicated otherwise in a credit line to the material. If material is not included in the article's Creative Commons licence and your intended use is not permitted by statutory regulation or exceeds the permitted use, you will need to obtain permission directly from the copyright holder. To view a copy of this licence, visit http://creativecommons. org/licenses/by/4.0/.

Publisher's Note Springer Nature remains neutral with regard to jurisdictional claims in published maps and institutional affiliations. 